\title{
Structure, Microstructure and Optical Absorption Analysis of CuO Nanoparticles Synthesized by Sol-Gel Route
}

\author{
P. Mallick* ${ }^{*}$ S. Sahu \\ Department of Physics, North Orissa University, Baripada, 757003, India \\ pravanjan_phy@yahoo.co.in
}

\begin{abstract}
We report the synthesis of $\mathrm{CuO}$ nanoparticles using different solvents by a low cost sol-gel route. Evolution of structure, microstructure and optical absorption analysis of these nanoparticles were studied using X-ray diffraction (XRD) and UV-Visible spectrophotometer. XRD analysis indicated that the crystallite size and strain are higher for the $\mathrm{CuO}$ nanoparticle synthesized using propanol as solvent. Optical absorption analysis indicated the red shift of indirect band gap and the blue shift of direct band gap. In the present case, red shift is associated with the formation of surface defects whereas the blue shift is due to the quantum confinement effect seen for nanoparticle systems.
\end{abstract}

Keywords Transition Metal Oxide, Sol-Gel Route, Nanoparticle, Cuo

\section{Introduction}

In recent years, nanoscale metal oxides have attracted a great deal of research interest because of both fundamental and technological point of view. Among all the metal oxides, cupric oxide $(\mathrm{CuO})$ has attracted considerable attention because of its peculiar properties. $\mathrm{CuO}$ has been used as a basic material in cuprate High- $\mathrm{T}_{\mathrm{C}}$ superconductors as the superconductivity in these classes of systems is associated with $\mathrm{Cu}-\mathrm{O}$ bondings[1,2]. Apart from this, $\mathrm{CuO}$ has investigated as potential material for nanofluid in heat transfer applications[3], catalysts for the water-gas shift reaction[4], steam reforming [5], CO oxidation of automobile exhaust gases[6], photocathodes for photoelectrochemical water splitting application[7] etc. For technological applications the detailed understanding of size, morphology controlled emergence of different properties are important.

The synthesis procedure plays crucial role in controlling the size, shape of the nanostructure and hence detecting different properties of the material. $\mathrm{CuO}$ nanoparticles have been prepared by wet-chemistry route[8], sonochemical preparation[9], alkoxidebased preparation[10], hydrothermal process[11], solid-state reaction in the presence of a surfac$\operatorname{tant}[12]$ etc.

In the present study, we have synthesized $\mathrm{CuO}$ nanoparticles using different solvent by a low cost sol-gel process. The aim of the present paper is to study the effect of different

* Corresponding author:

pravanjan_phy@yahoo.co.in (P. Mallick)

Published online at http://journal.sapub.org/nn

Copyright (C) 2012 Scientific \& Academic Publishing. All Rights Reserved solvent on the structure, microstructure and optical band gap of $\mathrm{CuO}$ synthesized by sol-gel route.

\section{Experimental Methods}

For the synthesis of $\mathrm{CuO}$ nanoparticles in sol-gel process, $5 \mathrm{gm}$ of $\mathrm{Cu}\left(\mathrm{NO}_{3}\right)_{2 .} . \mathrm{H}_{2} \mathrm{O}$ is dissolved into $20 \mathrm{ml}$ of ethanol. In order to see the effect of solvent, we use propanol instead of ethanol for the synthesis of another $\mathrm{CuO}$ nanoparticles. $\mathrm{Cu}\left(\mathrm{NO}_{3}\right)_{2} \cdot \mathrm{H}_{2} \mathrm{O}$ dissolved in two different solvents separately stirred for 1 hour to obtained for the homogenious solutions. These solutions kept for 1 day for gel formation. Then the gels were dried at $200^{\circ} \mathrm{C}$ and calcined at $300^{\circ} \mathrm{C}$ for 1 hour in each step. Then the obtained powders pressed into pellets. Finally these pellets were annealed at $500^{\circ} \mathrm{C}$ for 1 hour.

The structural and optical properties of the $\mathrm{CuO}$ nanoparticles were studied by using Bruker X-ray diffractometer (D8 Advance) and UV-Visible spectrophotometer (Simadzu, UV-2450) respectively.

\section{Results and Discussion}

\subsection{Structure and Microstructure of $\mathrm{CuO}$ Nanoparticles}

Figure 1 shows the XRD pattern of $\mathrm{CuO}$ nanoparticles synthesized by sol-gel route using ethanol and propanol as solvent. In both solvent cases all the obtained peaks in the XRD pattern are well matched with the monoclinic phase of $\mathrm{CuO}$ bulk crystals and well consistent with the JCPDS card (card no: 048-1548). No impurity peak related to any other phases of $\mathrm{Cu}$ like $\mathrm{Cu}(\mathrm{OH})_{2}, \mathrm{Cu}_{2} \mathrm{O}$ or $\mathrm{Cu}$ are seen in the ob- 
served XRD pattern. Our XRD results thus confirm synthesis of pure and well crystalline $\mathrm{CuO}$ nanoparticles without any impurity. The obtained results are well consistent with the previously reported literature[13,14]. The XRD peaks broaden and shift to higher angles for the $\mathrm{CuO}$ sample prepared when propanol used as solvent. The peak shift could be due to strain generation in the materials medium during synthesis. Since two different batches $\mathrm{CuO}$ nanoparticles were synthesized using ethanol and propanol as solvent. The solvent might be influencing the microstructure of resultant $\mathrm{CuO}$ nanoparticle.

In order to understand the peak shift for $\mathrm{CuO}$ nanoparticle synthesized by both solvent cases, we carried out Williamson-Hall (W-H) analysis[15] of the FWHM $(\beta)$ of various Bragg peaks appeared in the XRD pattern (Fig. 1). The W-H plot of $\frac{\beta \cos \theta}{\lambda}$ versus $\frac{2 \sin \theta}{\lambda}$ gives the value of microstrain from the slope and particle size from the ordinate intersection. For pure particle size broadening this plot is expected to be a horizontal line parallel to the $\sin \theta$ axis, whereas in the presence of strain, it has a non-zero slope. Figure 2 shows the W-H plot for $\mathrm{CuO}$ nanoparticle synthesized with two different solvents. The obtained values of crystallite size and microstrain for $\mathrm{CuO}$ nanoparticles synthesized under different solvent are given in Table 1. As indicated from the Table 1, the crystallite size and strain is higher for the $\mathrm{CuO}$ nanoparticle synthesized using propanol as solvent. The shifting of XRD peaks to higher angle may be a consequence of stain effect.

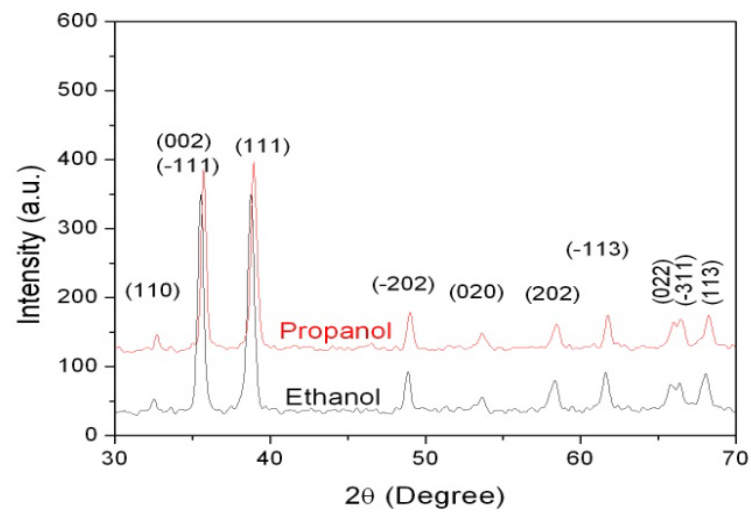

Figure 1. X-ray diffraction pattern of $\mathrm{CuO}$ nanoparticles synthesized by sol-gel route with different solvent as mentioned

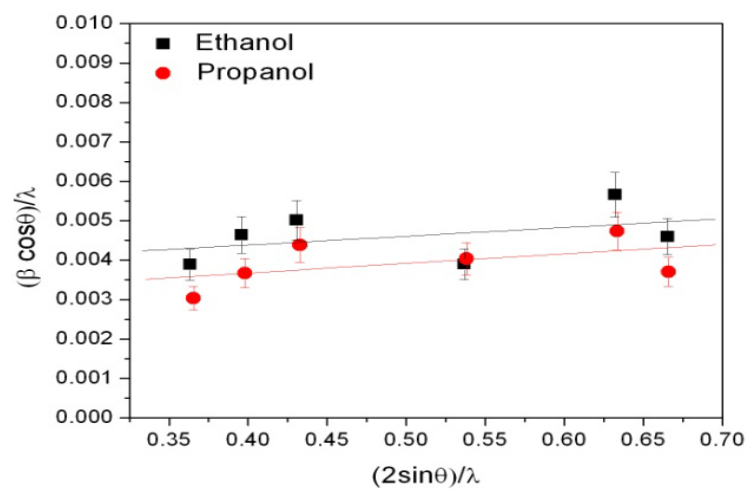

Figure 2. Williamson-Hall (W-H) plot for the $\mathrm{CuO}$ nanoparticles synthesized by sol-gel route with different solvent as mentioned
Table 1. Evolution of crystallite size and strain of $\mathrm{CuO}$ nanoparticles synthesized with different solvent as mentioned

\begin{tabular}{|c|c|c|}
\hline Solvent & Crystallite Size (nm) & Strain (\%) \\
\hline Ethanol & 28.57 & 0.22 \\
\hline Propanol & 36.76 & 0.24 \\
\hline
\end{tabular}

\subsection{UV-Visible Characterization of $\mathrm{CuO}$ Nanoparticle}

The variation of absorption coefficient, $\alpha$ of $\mathrm{CuO}$ nanoparticles as a function of wavelength is shown in Fig. 3. It is clearly seen from the figure that the absorption coefficient tends to decrease exponentially as the wavelength increases. This behaviour is typical for many semiconductors and can occur for a variety of reasons, such as internal electric fields within the crystal, deformation of lattice due to strain caused by imperfection and inelastic scattering of charge carriers by phonons[16-18]. The absorbance of $\mathrm{CuO}$ sample synthesized with propanol solvent shows faster exponential decrease indicating more strain generation in this case. The behaviour of absorbance shown in Fig. 3 is thus agreed with the strain analysis using W-H plot discussed above.

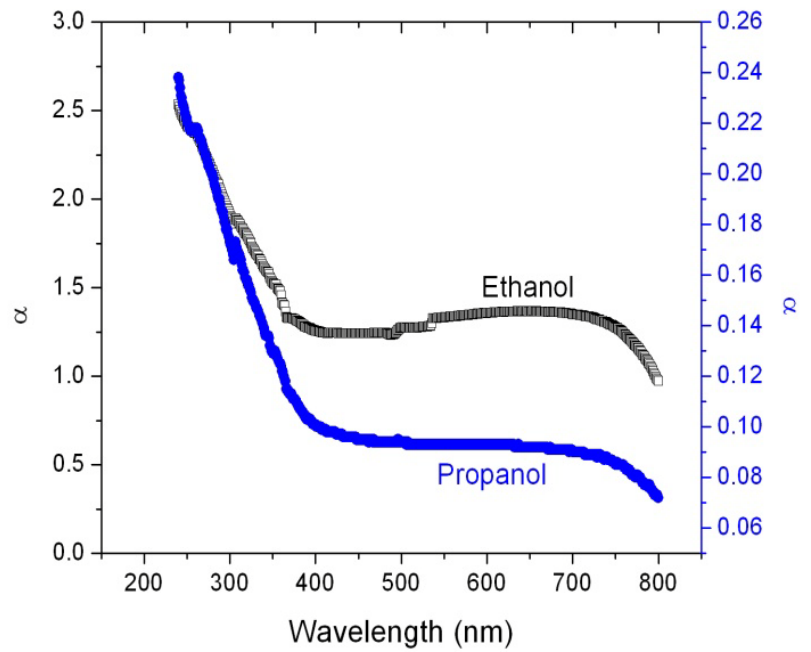

Figure 3. Variation of absorption coefficient of $\mathrm{NiO}$ nanoparticles as a function of wavelength

The optical band gap of $\mathrm{CuO}$ nanoparticles were extracted according to the following relation[19]:

$$
\alpha=\frac{B\left(h v-E_{g}\right)^{n}}{h v}
$$

where $h v$ is the incident photon energy, $\alpha$ is the absorption coefficient, $B$ is a materials dependent constant and $E_{g}$ is the optical band gap. The value of $n$ depends on the nature of transition. Depending on whether the transition is direct allowed, direct forbidden, indirect allowed or indirect forbidden, $n$ takes the value $1 / 2,3 / 2,2$ or 3 respectively[20]. The usual method of determining $E_{g}$ involves plotting $(\alpha h v)^{1 / n}$ vs. photon energy, $h v$. Figure 4 and 5 show the variation of $(\alpha h v)^{1 / n}$ vs. $h v$ for $\mathrm{CuO}$ nanoparticles with $n$ values of $1 / 2$ and 2 respectively. The values of direct and indirect band gap for $\mathrm{CuO}$ nanoparticles synthesized with different solvent are shown in Table 2. The indirect band gap of $\mathrm{CuO}$ nanoparticles synthesized using both 
the solvents show similar values and the values red shifted 0.24 to $0.27 \mathrm{eV}$ as compared to bulk value $(1.45 \mathrm{eV})[21]$. The increasing red shift with decreasing particle size suggests that the defects responsible for the intra-gap states are primarily of surface defects[22-24]. Our results thus indicated that $\mathrm{CuO}$ nanoparticles prepared using ethanol as solvent show more surface defects as compared to the $\mathrm{CuO}$ nanoparticles prepared using propanol as solvent. Both the $\mathrm{CuO}$ samples show higher direct band gap as compared to bulk value $(3.25 \mathrm{eV}[24,25])$. The blue shift in the direct band edges as seen in present case is due to the quantum confinement effect[24,26].

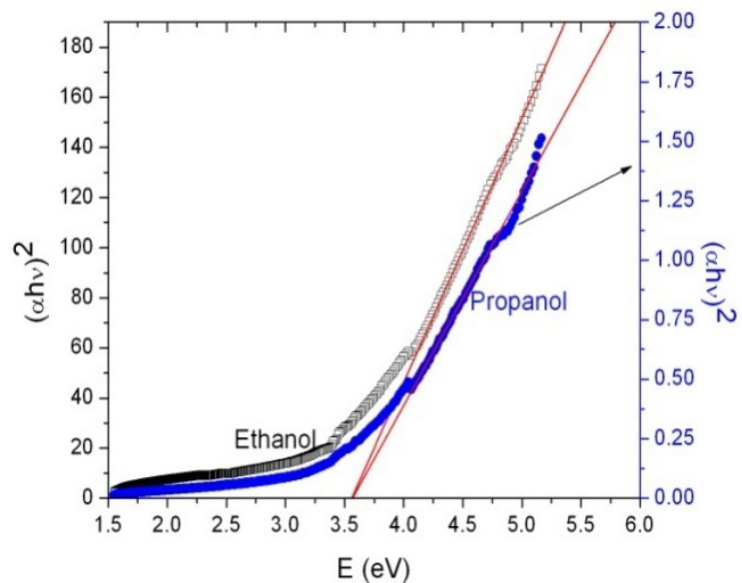

Figure 4. Variation of $(\alpha h v)^{2}$ vs. photon energy, $h v$ for $\mathrm{CuO}$ nanoparticles prepared sol-gel route with different solvent as mentioned

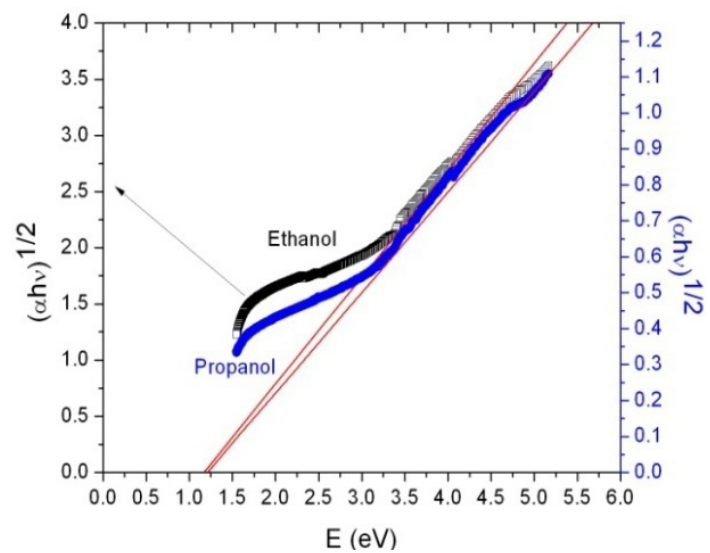

Figure 5. Variation of $(\alpha h v)^{1 / 2}$ vs. photon energy, $h v$ for $\mathrm{CuO}$ nanoparticles prepared sol-gel route with different solvent as mentioned

Table 2. Evolution of direct and indirect optical band gap of $\mathrm{CuO}$ nanoparticles synthesized with different solvent as mentioned

\begin{tabular}{|c|c|c|}
\hline \multirow{2}{*}{ Solvent } & \multicolumn{2}{|c|}{ Optical band gap (eV) } \\
\cline { 2 - 3 } & Direct & Indirect \\
\hline Ethanol & 3.57 & 1.18 \\
\hline Propanol & 3.57 & 1.21 \\
\hline
\end{tabular}

\section{Conclusions}

$\mathrm{CuO}$ nanoparticles were synthesized by a low cost sol-gel method. Effect of solvent on the structure, microstructure and optical absorption properties of $\mathrm{CuO}$ nanoparticles were studied. XRD analysis indicated that the crystallite size and strain are higher for the $\mathrm{CuO}$ nanoparticle synthesized using propanol as solvent. UV-Visible analysis also indicated the higher strain generation for $\mathrm{CuO}$ nanoparticle synthesized using propanol as solvent. Optical absorption analysis indicated that the both the $\mathrm{CuO}$ samples show red shift of indirect band gap due to the formation of surface defects. $\mathrm{CuO}$ nanoparticles on the other hand show higher direct band gap as compared to bulk value indicating blue shift of band gap due to the quantum confinement effect.

\section{REFERENCES}

[1] F. Parmigiani and G. Samoggia, "Experimental Evidence of a Fluctuating Charge State in Cupric Oxide", Europhys. Lett. 7, 543(1988).

[2] X.G. Zheng, C.N. Xu, Y. Tomokiyo, E. Tanaka, H. Yamada and Y. Soejima, "Observation of Charge Stripes in Cupric Oxide", Phys. Rev. Lett. 85, 5170 (2000).

[3] M.-H. Chang, H.-S. Liu and C.Y. Tai, "Preparation of copper oxide nanoparticles and its application in nanofluid", Powder Technol. 207, 378 (2011).

[4] Y. She, Q. Zheng, L. Li, Y. Zhan, C. Chen, Y. Zheng and $\mathrm{X}$.Lin, "Rare earth oxide modified $\mathrm{CuO} / \mathrm{CeO}_{2}$ catalysts for the water-gas shift reaction", Int. J. Hydrogen Energy 34, 8929 (2009).

[5] P.P.C. Udani, P.V.D.S. Gunawardana, H.C. Lee, D.H. Kim, "Steam reforming and oxidative steam reforming of methanol over $\mathrm{CuO}-\mathrm{CeO}_{2}$ catalysts", Int. J. Hydrogen Energy 34, 7648 (2009).

[6] J.L. Cao, G.S. Shao, Y. Wang, Y. Liu and Z.Y. Yuan, "CuO catalysts supported on attapulgite clay for low-temperature CO oxidation", Catal. Commun. 9, 2555 (2008)

[7] C.-Y. Chiang, K. Aroh, N. Franson, V.R. Satsangi, S. Dass and S. Ehrman, "Copper oxide nanoparticle made by flame spray pyrolysis for photoelectrochemical water splitting Part II. Photoelectrochemical study", Int. J. Hydrogen Energy 36, 15519 (2011).

[8] X.P. Gao, J.L. Bao and G.L. Pan, "Preparation and Electrochemical Performance of Polycrystalline and Single Crystalline $\mathrm{CuO}$ Nanorods as Anode Materials for Li Ion Battery”, J. Phys. Chem. B 108, 5547 (2004).

[9] R. Vijaya Kumar, R. Elgamiel, Y. Diamant, and A. Gedanken, "Sonochemical Preparation and Characterization of Nanocrystalline Copper Oxide Embedded in Poly(vinyl alcohol) and Its Effect on Crystal Growth of Copper Oxide", Langmuir 17, 1406 (2001).

[10] C.L. Carnes, J. Stipp and K.J. Klabunde, "Synthesis, Characterization, and Adsorption Studies of Nanocrystalline Copper Oxide and Nickel Oxide", Langmuir 18, 1352 (2002).

[11] Y. Zhang, S. Wang, X. Li, L. Chen, Y. Qian and Z. Zhang, " $\mathrm{CuO}$ shuttle-like nanocrystals synthesized by oriented attachment”, J. Cryst. Growth 291, 196 (2006). 
[12] W. Wang, Y. Zhan and G. Wang, "One-step, solid-state reaction to the synthesis of copper oxide nanorods in the presence of a suitable surfactant", Chem. Commun. 727 (2001).

[13] J.I. Langford and D. Louer, "High-resolution powder diffraction studies of copper(II) oxide", J. Appl. Crystallogr. 24, 149 (1991).

[14] M. Abaker, A. Umar, S. Baskoutas, S.H. Kim and S.W. Hwang, "Structural and optical properties of $\mathrm{CuO}$ layered hexagonal discs synthesized by a low-temperature hydrothermal process", J. Phys. D: Appl. Phys. 44, 155405 (2011).

[15] G.K. Williamson and W.H. Hall, "X-ray line broadening from filed aluminium and wolfram", Acta Metall. 1, 22 (1953).

[16] T.S. Moss, G.J. Burrell and B. Ellis, Semiconductor Opto-Electronics, Butterworth \& Co. Ltd, 1973.

[17] H.M. Honsi, S.A. Fayek, S.M. El-Sayed, M. Roushdy, M.A. Soliman, "Optical properties and DC electrical conductivity of $\mathrm{Ge}_{28-\mathrm{x}} \mathrm{Se}_{72} \mathrm{Sb}_{\mathrm{x}}$ thin films", Vacuum 81, 54 (2006)

[18] A. Sawaby, M.S. Selim, S.Y. Marzouk, M.A. Mostafa and A. Hosny, "Structure, optical and electrochromic properties of $\mathrm{NiO}$ thin films", Physica B 405, 3412 (2010).

[19] N.F. Mott, E.A. Davies, Electronic Processes in Non-Crystalline Materials (Clarendon Press, Oxford,1979).
[20] A.N. Banerjee and K.K. Chattopadhyay, in D. Depla and S. Maheiu (Eds.), Reactive Sputter Deposition, Springer-Verlag Berlin Heidelberg, 2008, p.465.

[21] B.A. Gizhevskii, Y.P. Sukhorukov, A.S. Moskvin, N.N. Loshkareva, E.V. Mostovshchikova, A.E. Ermakov, E.A. Kozlov, M.A. Uimin, V.S. Gaviko, "Anomalies in the optical properties of nanocrystalline copper oxides $\mathrm{CuO}$ and $\mathrm{Cu} 2 \mathrm{O}$ near the fundamental absorption edge", JETP 102, 297(2006).

[22] Y.P. Sukhorukov, B.A. Gizhevskii, E.V. Mostovshchikova, A.Y. Yermakov, S.N. Tugushev and E.A. Kozlov, "Nanocrystalline copper oxide for selective solar energy absorbers", Tech. Phys. Lett. 32, 132 (2006).

[23] S.G. Ovchinnikov, B.A. Gizhevskii, Y.P. Sukhorukov, A.E. Ermakov, M.A. Uimin, E.A. Kozlov, Y. Kotov and A.A.V. Bagazeev, "Specific features of the electronic structure and optical spectra of nanoparticles with strong electron correlations", Phys. Solid State 49, 1116 (2007).

[24] S. Rehman, A. Mumtaz and S.K. Hasanain, "Size effects on the magnetic and optical properties of $\mathrm{CuO}$ nanoparticles", J. Nanopart. Res. 13, 2497 (2011).

[25] F.P. Koffyberg and F.A. Benko, "A photoelectrochemical determination of the position of the conduction and valence band edges of p - type C u0 ”, J. A ppl. Phys. 53, 1173 (1982).

[26] S. Neeleshwar, C.L. Chen, C.B. Tsai, Y.Y. Chen, C.C. Chen, S.G. Shyu and M.S. Seehra, "Size-dependent properties of CdSe quantum dots”, Phys. Rev. B 71, 201307(R) (2005). 